\title{
Multifrequency acoustics as a probe of mesoscopic blood coagulation dynamics
}

\author{
Adarsh Ganesan ${ }^{1}$, Gokulnath Rajendran ${ }^{2}$, Ari Ercole $^{2 *}$, Ashwin Seshia $^{1 * \$}$ \\ ${ }^{1}$ Nanoscience Centre, University of Cambridge, Cambridge CB3 OFF, UK. \\ ${ }^{2}$ Division of Anaesthesia, University of Cambridge, Cambridge CB2 0QQ, UK. \\ "These authors contributed equally to the supervision of this project \\ ${ }^{\$}$ Corresponding author
}

Coagulation is a complex enzymatic polymerisation cascade. Disordered coagulation is common in medicine and may be life-threatening yet clinical assays are typically bulky and/or provide an incomplete picture of clot mechanical evolution. We present the adaptation of an in-plane acoustic wave device: quartz crystal microbalance with dissipation (QCM-D) at multiple harmonics to determine the time-evolution of mesoscale mechanical properties of clot formation in vitro. This approach is sensitive to changes in surface and bulk clot structure in various models of induced coagulopathy. Furthermore, we are able to show that clot formation at surfaces has different kinetics and mechanical strength to that in the bulk, which may have implications for the design of bioprosthetic materials. The 'Multifrequency acoustics' approach thus enables unique capability to portray biological processes concerning blood coagulation.

Compound soft systems such as polymer solutions, melts, emulsions, gels and foams form a peculiar type of condensed matter where viscoelastic properties are controlled by the spatial arrangement of component filaments [1-6]. Coagulating blood is a highly complex heterogeneous, spatially and kinetically variable polymerising soft-system. In the face of initiation by injury, a complex system of prothrombotic enzymatic and cellular processes must rapidly propagate a clot by autocatalytic localised polymerisation and cellular interactions [7]. The mechanical properties of the clot are determined by the microstructural configuration and must be sufficiently strong to arrest bleeding [8]. Furthermore, a polymer degradation ("fibrinolysis") pathway ensures that the clot does not persist inappropriately once tissue has been repaired. These competing processes and ultimate clot microstructure are exquisitely sensitive to initiation conditions and yet must be both quantitatively balanced and correctly temporally sequenced. Conventional laboratory assays for characterising coagulation disorders are based on primitive measurements of gelation time after clot initiation and poorly reflect in vivo coagulation kinetics and mechanical properties. More recent rheological methods such as thromboelastography (TEG) [9] measure clot strength but operate at clot mechanical shears that are not physiological and are 
sensitive to millimetre length-scales which are far longer than the characteristic scales of clot microstructure. All of the current assays are flawed and so a better quantitative spatio-temporal characterization sensitive to mesoscopic clot properties would be of great clinical interest in diagnosis, resuscitation and in monitoring anticoagulation therapies. Furthermore, improved understanding of the interaction of coagulating blood and artificial surfaces can allow physicochemical design of materials with desirable pro- or antithrombotic surface properties for implant application.

Here we attempt to characterize blood coagulation using information extracted from the Quartz Crystal Microbalance with Dissipation (QCM-D). QCM-D is an acoustic wave device and has been demonstrated to be sensitive to coagulation previously [10-14]. We extend the use of QCM-D to probe the coagulation using multiple harmonics of in-plane thickness-shear vibrations. The acoustic penetration depths in blood resulting from these in-plane waves depends on frequency and is of the order of $100 \mathrm{~nm}$ and therefore comparable to the length of the fibrin monomer (around 40nm). Thus the acoustic probe presents a unique sensitivity to morphologically relevant mesoscopic length-scales which may be varied by studying the acoustic properties at different frequencies. To this end we modified the existing theory [15] to extract depth-dependent physical properties from the frequency dependence of QCM-D responses.

Our QCM-D based mesoscopic characterization technique is based on the classical Stokes' second flow problem that allows the quartz resonator to be just sensitive to a tiny region next to its surface (in the order of $100 \mathrm{~nm}$ for frequencies in tens of $\mathrm{MHz}$ and fluidic properties similar to water). As the fibrin clots, the frequency and energy dissipation of quartz resonant modes get modulated depending on the spatial distribution of clot density and rigidity in blood during clot organisation. We use the frequency dependent QCM-D technique to track depth dependent properties as a function of time for coagulation and fibrinolysis during natural and artificial conditions. The shifts in resonant frequency and energy dissipation of thickness shear waves due to the evolving physical properties of clot are given by [16]

$$
\begin{gathered}
\Delta f(\delta)=-\alpha_{0} \frac{\rho}{\delta}-\alpha_{1} \frac{\Delta \eta_{l}}{\delta}+\alpha_{2} E \delta \\
\Delta D(\delta)=\beta_{0} \rho \delta+\beta_{1} \Delta \eta_{l} \delta+\beta_{2} E \delta^{3}
\end{gathered}
$$

where $E$ and $\rho$ represent shear modulus and density of clot; $\kappa$ is the shear stiffness between clot and quartz surface; $\delta$ is the Stokes length which is given by $\delta=\sqrt{\eta_{l} /\left(\rho_{l} \pi f\right)}$ where $\rho_{l}$ and $\eta_{l}$ are density and 
viscosity of plasma. Using multi-frequency measurement, it is thus possible to track the underlying depth $(\delta)$-dependent physical changes depending on trends observed in experiments.

During coagulation on the QCM surface, there are fundamentally surface effects (including adhesion resulting from physicochemical interactions and surface initiation induced by enzymatic processes) and bulk effects (predominantly enzymatic). For the experimental validation of our method, four representative coagulation cascades exhibiting different degrees of surface and bulk effects during clot formation and lysis are considered viz. 1. 'Initiated coagulation' (representing 'normal' tissue-factor induced coagulation); 2. 'Hyperfibrinolysis' (an inappropriate activation of the fibrinolytic pathway that has been recently described after major haemorrhage but to which only TEG is sensitive); 3 . 'Antagonised hyperfibrinolysis' (representing the situation of treated hyperfibrinolysis and demonstrating the reversibility of changes to reversal of fibrinolysis) and 4. 'Antagonised intrinsic fibrinolysis' [16]. Condition 4 is of particular interest- there is evidence that administration of antifibrinolytic drugs improve survival after major haemorrhage but this must be given early [17]- the majority of such patients do not have a disorder of fibrinolysis as measured by conventional assays and therefore a mechanism for the effect of this drug in this context is unclear. Figure $1 \mathrm{~B}$ and $1 \mathrm{C}$ shows the corresponding acoustic responses of QCM-D. Overall, the loading of QCM-D due to fibrin polymerization and clot solidification decreases the resonant frequency and increases the dissipation with time as previously reported in the literature [10-14]._During this measurement, temperature effects are not significant [16]. Hence, the modulation of acoustic responses can be solely attributed to the density and rigidity changes during clotting. Also, the other parameters such as surrounding liquid viscosity and pore size are only seen as correction factors for the absolute density of clot. That is, the clot density termed here, represents a collective average of actual density and pore size of clot and surrounding liquid viscosity. Using eq. (1), the temporal trends of clot density and rigidity that provide a measure of the complex interplay of physical processes are extracted [16].

In our experimental conditions that mimic the standard prothrombin time assay, tissue factor is present in excess and a phase change from liquid to gel is seen within $<15$ seconds). However it is clear that mechanical changes occur at longer timescales than this phase change presumably due to on-going crosslinking processes. Although the coagulation is 'Tissue-factor initiated' [16], our study provides an evidence for a dominant contact activation pathway in the mesoscopic region (Figure 2A) (cf [18]). This suggests that surface initiation is an important determinant of clot mechanical structure. Consequently, the clots formed near inert 2D gold surfaces have higher density. 
In contrast to 'Initiated coagulation', the 'antagonised intrinsic fibrinolysis' case exhibits a spatially homogenous clot density in the bulk (Figure 2C). Intrinsic fibrinolysis is important, from the outset, in determining bulk clot density. Conventional clinical wisdom regards fibrinolysis as a late phenomenon related to clot breakdown [8], something that conventional assays have not been sensitive to. Our result suggests a mechanism for the beneficial effects of early administration of antifibrinolytics [17] even in patients in whom hyperfibrinolysis would not yet have occurred. Whilst states of uncontrolled hyperfibrinolysis do occur clinically with devastating effects on survival, these are relatively rare- for example after massive haemorrhage or transfusion. It is known administration of TxA to bleeding trauma patients [17] confers a survival benefit but this is only seen when given early- suggesting an effect that does not depend on the development of a hyperfibrinolytic state which occurs later and then only in a subset of patients. An effect of intrinsic fibrinolysis on early clot stability is therefore a plausible explanation for this and our assay is sensitive to such a process unlike conventional methods. Since antifibrinolytic therapy may also be associated with adverse outcome such as thromboembolic events that may be devastating, a more refined understanding of the role of fibrinolysis in the determination of clot structure is timely. In addition to antifibrinolytic effect, TxA may have complex dose-dependent effects on mesoscopic clot property kinetics (Figure 2D).

During hyperfibrinolysis, the clot is broken down by tPA. This seems to be broadly similar to TEG. Interestingly, compared to figure $2 \mathrm{~A}$, even with hyperfibrinolysis, bulk clot density is higher. It is recognised that tPA may have some paradoxical early prothrombotic effects [19] which may explain our results although conventional clinical assays are not sensitive to this. Our study also suggests this early positive effect on bulk clot density during hyperfibrinolysis. Figure $2 \mathrm{~F}$ also suggests that the hyperfibrinolysis is also involved in determining the stiffness of the interface properties. Presumably the interfacial layer becomes closer to the surface. Finally, the figure $2 \mathrm{G}$ shows that the 'antagonised hyperfibrinolysis' is just an intermediate between normal and hyperfibrinolysis indicating a partial reversal by TXA.

In all of the coagulation studies, high rigidity surface (2D) layers and low rigidity bulk (3D weak structure) are associated with the clot microstructure. Further there is also evidence for a very stiff intermediate interlayer which has not been previously described. We propose this to be due to a transition region between surface (2D constrained) and bulk (free) polymerization structures (Figure 3).

This technique clearly provides information that qualitatively replicates TEG but in a more robust and compact form-factor without macroscopically moving parts. Additionally, the sensitivity to surface and 
bulk properties as a function of time give further information about clot mesoscopic properties and dynamics of clot formation as well as the interplay between multiple pro/antithrombotic pathways, interface effects and fibrinolysis. It is in principle straightforward to modify the QCM-D surface both chemically and physically (e.g. by nanoscale patterning) to obtain more insights into the physics of coagulation. The surface sensitivity of our method will prove useful in designing surface coatings with bespoke biological properties for example for bioprostheses. Further, the technique can be harnessed for evaluating the biocompatibility and toxicity of different materials to blood coagulation. Also, the lifetime of device utilizing our technique (such as QCM-D in our case) needs to be improved for practical application.

This paper introduces a QCM-D based experimental technique to probe mesoscopic dynamics of blood coagulation. This study can be extended by probing the perturbations of higher harmonics ( $>7$ ) for exploring the interface in greater detail. Our scalable technique can be readily implemented in tiny devices that are realized with custom acoustic properties using microfabrication and thin-film processing technologies. The underlying molecular level processes in the measured mesoscopic properties can also be studied through intense theoretical efforts. In this way, the technique has potential for the investigation of time-varying surface dependent mechanical effects in complex systems including protein aggregation and poly-gelation. For real world applications, universal to any metrology, the parameter being measured using our experimental technique based on QCM-D has to suffer minimal influence from the other parameters.

Funding from the Cambridge University Engineering for Clinical Practice initiative and the Cambridge Trusts is gratefully acknowledged. AG would like to thank Dr Jerome Charmet and Dr Abhinav Prasad for their initial contributions towards the experimental setup. AE would like to thank Dr Trevor Baglin for useful discussions.

\section{References}

[1] D. Humphrey, C. Duggan, D. Saha, D. Smith, and J. Kas, "Active fluidization of polymer networks through molecular motors," Nature 416 (6879), 413-416 (2002).

[2] Tanniemola B. Liverpool and M. Cristina Marchetti, "Rheology of active filament solutions," Physical review letters 97 (26), 268101 (2006).

[3] D. Marenduzzo, E. Orlandini, and J. M. Yeomans, "Hydrodynamics and rheology of active liquid crystals: A numerical investigation," Physical review letters 98 (11), 118102 (2007). 
[4] Luca Giomi, Tanniemola B. Liverpool, and M. Cristina Marchetti, "Sheared active fluids: Thickening, thinning, and vanishing viscosity," Physical Review E 81 (5), 051908 (2010).

[5] S. Q. Choi, S. Steltenkamp, J. A. Zasadzinski, and T. M. Squires, "Active microrheology and simultaneous visualization of sheared phospholipid monolayers," Nat Commun 2, 312 (2011).

[6] E. J. Hemingway, A. Maitra, S. Banerjee, M. C. Marchetti, S. Ramaswamy, S. M. Fielding, and M. E. Cates, "Active Viscoelastic Matter: From Bacterial Drag Reduction to Turbulent Solids," Physical review letters 114 (9), 098302 (2015).

[7] Maureane Hoffman, "A cell-based model of coagulation and the role of factor VIla," Blood reviews 17, S1-S5 (2003).

[8] John W. Weisel, "The mechanical properties of fibrin for basic scientists and clinicians," Biophysical chemistry 112 (2), 267-276 (2004).

[9] Luis Teodoro da Luz, Bartolomeu Nascimento, and Sandro Rizoli, "Thrombelastography (TEGÂA $\hat{}^{\circledast}$ : practical considerations on its clinical use in trauma resuscitation," Scand J Trauma Resusc Emerg Med 21, 29 (2013).

[10] Tzong-Jih Cheng, Hsein-Chang Chang, and Tsun-Mei Lin, "A piezoelectric quartz crystal sensor for the determination of coagulation time in plasma and whole blood," Biosensors and Bioelectronics 13 (2), 147-156 (1998).

[11] G. Hayward, R. Dutton, and M. Young, "Thickness shear mode acoustic assay for plasminogen activators," Analytical Communications 35 (1), 25-28 (1998).

[12] Lothar Muller, S. Sinn, H. Drechsel, C. Ziegler, H.-P. Wendel, H. Northoff, and F. K. Gehring, "Investigation of prothrombin time in human whole-blood samples with a quartz crystal biosensor," Analytical Chemistry 82 (2), 658-663 (2009).

[13] Stefan Sinn, Lothar Muller, H. Drechsel, M. Wandel, H. Northoff, G. Ziemer, H. P. Wendel, and F. K. Gehring, "Platelet aggregation monitoring with a newly developed quartz crystal microbalance system as an alternative to optical platelet aggregometry," Analyst 135 (11), 2930-2938 (2010).

[14] Munawar Hussain, Hinnak Northoff, and Frank K. Gehring, "QCM-D providing new horizon in the domain of sensitivity range and information for haemostasis of human plasma," Biosensors and Bioelectronics 66, 579-584 (2015). 
[15] Stephen J. Martin, Victoria Edwards Granstaff, and Gregory C. Frye, "Characterization of a quartz crystal microbalance with simultaneous mass and liquid loading," Analytical Chemistry 63 (20), 22722281 (1991).

[16] See supplemental material at [URL will be inserted by AIP] for the model derivation, data extraction scheme and experimental methods.

[17] CRASH-2 collaborators, Roberts I, Shakur H, Afolabi A, Brohi K, Coats T, Dewan Y, Gando S, Guyatt G, Hunt BJ, Morales C, Perel P, Prieto-Merino D, Woolley $\quad T$. "The importance of early treatment with tranexamic acid in bleeding trauma patients: an exploratory analysis of the CRASH-2 randomised controlled trial." Lancet 377 (9771), 1096-101 (2011).

[18] X. Cheng, K. W. Putz, C. D. Wood, and L. C. Brinson, "Characterization of Local Elastic Modulus in Confined Polymer Films via AFM Indentation," Macromolecular rapid communications 36 (4), 391-397 (2015).

[19] K. A. Shuster, S. K. Wrobleski, A. E. Hawley, B. R. Lucchesi, D. R. Sorenson, I. L. Bergin, R. E. Sigler, K. E. Guire, M. H. Nowland, and T. W. Wakefield, "Prothrombotic effects of thrombolytic therapy in a rat (Rattus norvegicus) model of venous thrombolysis," Comparative medicine 63 (3), 244 (2013). 

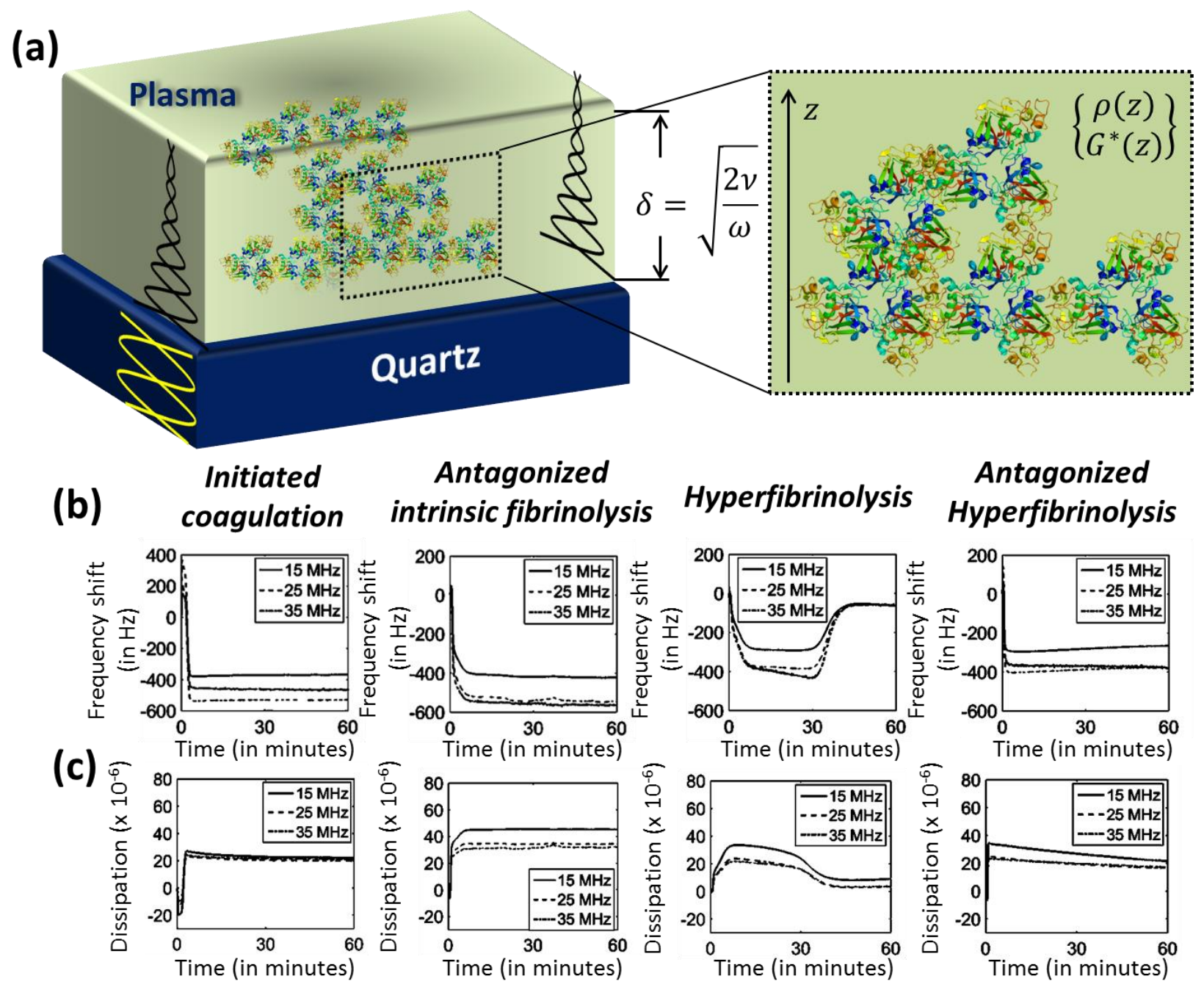

Figure 1: Scheme of acoustics aided spatial mapping: (a): Stokes flow is generated in the sample by the effect of thickness-shear vibrations of resonator. The corresponding damping length of this flow $(\delta)$ varies inversely with vibration frequency and therefore harmonic; (b) and (c): Resonant frequency shifts (panel (b)) and energy dissipation (panel (c)) against time for $3^{\text {rd }}, 5^{\text {th }}$ and $7^{\text {th }}$ harmonics $(15,25$ and $35 \mathrm{MHz}$ respectively) during coagulation under different conditions. Initiated coagulation (plasma + TF), hyperfibrinolysis (plasma+tPA+TF), antagonised hyperfibrinolysis (plasma + tPA + TXA + TF), antagonised intrinsic fibrinolysis (plasma + TxA + TF). 
(a) Initiated coagulation
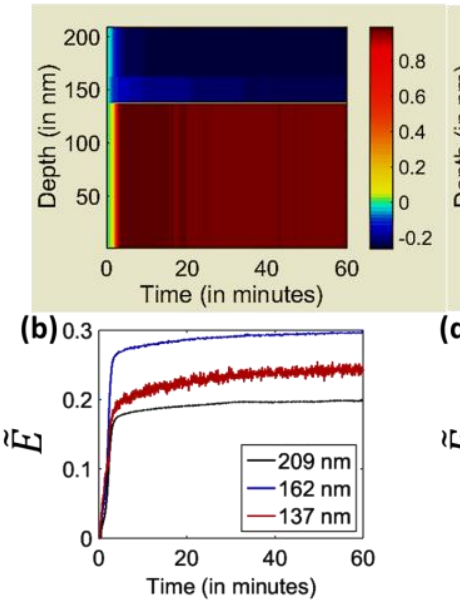

(c)

Antagonised intrinsic fibrinolysis

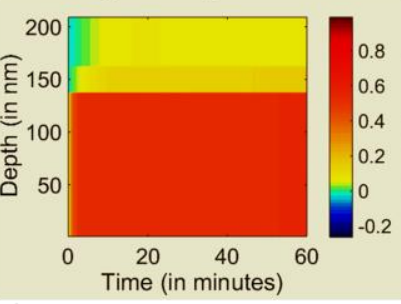

(d)

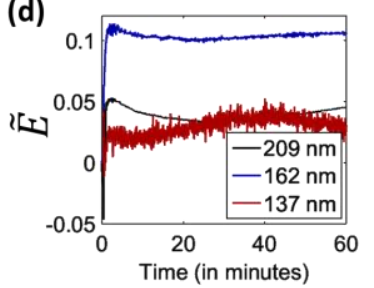

(e) Hyperfibrinolysis

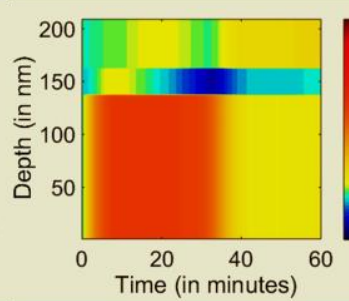

(f)

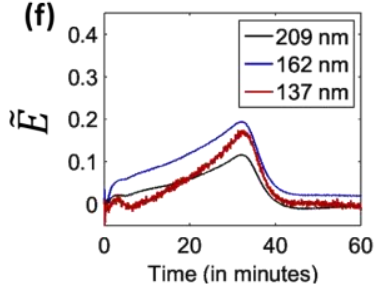

Antagonised

(g) hyperfibrinolysis

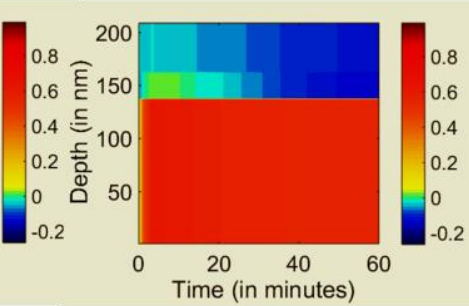

(h)

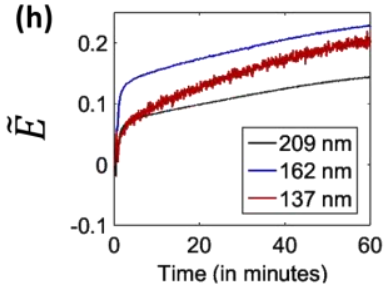

Figure 2: Spatio-temporal map of clot density and rigidity during coagulation: Change in fractional clot density $\left(\frac{\rho}{\rho_{l}}\right)$ (top panels) and fractional clot rigidity $\left(\frac{E}{\omega_{0} \eta_{l}}\right)$ (bottom panels) for corresponding to (a-b) initiated coagulation (plasma $+T F)$, (c-d) antagonised intrinsic fibrinolysis (plasma + TxA + TF), (e-f) hyperfibrinolysis (plasma + tPA + TF), (g-h) antagonised hyperfibrinolysis (plasma + tPA + TxA + TF) (Note: Green indicates baseline density in the figures $A, C, E$ and $G$ ). 


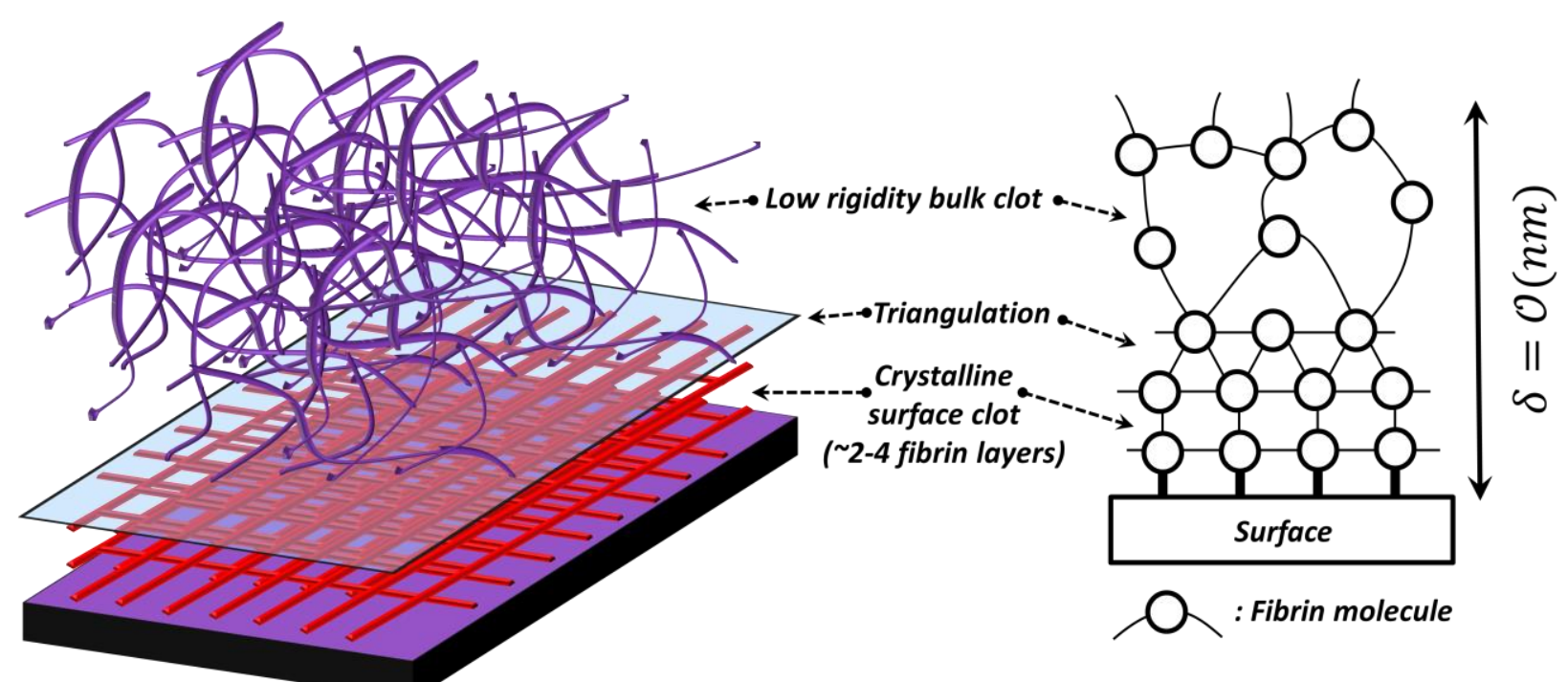

Figure 3: Cartoon representation of mesoscopic blood coagulation dynamics. Our results indicate a low rigidity bulk and intermediate rigidity surface layer are separated by an interlayer region of very high rigidity. We propose this to a result of the transition between 2D and 3D fibrin mesh properties. 